\title{
Harmonization and Homogenization on Fractals
}

\author{
Serguei M. Kozlov \\ Université de Provence, 3 place V. Hugo, F-13331 Marseille Cedex 3, France
}

Received March 26, 1992; in revised form October 30, 1992

\begin{abstract}
This paper suggests a direct approach to define the Laplacian, the spectral dimension of nested fractals and the pre-Sierpinski carpet conductivity. We find a geometric construction of the harmonic functions on the gasket and therefore can describe effectively the dense set of functions having finite energy. The paper is mostly aimed at the homogenization on the pre-Sierpinski gasket, whose horizontal and nonhorizontal bonds have different conductivities: $a$ and $b$ respectively. We prove the $\Gamma$-convergence of the rescaled energies on the pre-Sierpinski gasket to $\sigma(a, b) \varepsilon$, where $\varepsilon$ is the standard energy on the gasket with uniform conductivities. We also find an explicit expression for the effective conductivity $\sigma(a, b)$ and deduce that its set of singularities turns out to be the Julia set of a certain rational function. A special section is devoted to the problem of the pre-Sierpinski carpet conductivity asymptotic behavior; for this problem a new proof of Barlow-Bass inequalities with sharper constants is given.
\end{abstract}

\section{Introduction}

The fractals, the first example of which was given by Sierpinski [1] at the beginning of the century as an example of the set with the bizzare geometrical properties, were proposed more recently as models for different physical phenomena by Mandelbrot [2]. Then the Laplacian on the fractals and their spectral dimension which first appeared in the physical literature [2,3], see review [4]) as the tools of the investigation of the percolation effects and various transport processes, in classical as well as in quantum mechanics became the subject of intensive mathematical research [5-9]. Even in the case of fractals with uniform properties, and all the quoted papers devoted to that case, this subject is related to the theory of certain inhomogeneous media and has something in common with homogenization theory. At the same time the main assumption of that theory (which is in the most general case statistical translation invariance) is violated in the fractal case. In this paper we go further and, probably, for the first time, at least in the mathematical literature, 
discuss also the fractals with locally nonuniform properties. Fractal inhomogeneous geometry discussed here presents a new very interesting type of geometry where even the simplest disorder does not have the translation invariance property. However, this research was based on homogenization theory. Here we use such notions of this theory as $\Gamma$-convergence, harmonic coordinates and interchange duality [10-16]. Physically this approach means that we consider the pre-Sierpinski gasket as a resistor network of equal (Sect. 2) or two different types (Sect. 3) of resistors.

The purpose of Sect. 2 is to present a new, direct construction of the Laplacian on the Sierpinski gasket and a simple definition of its spectral dimension. It is a difficult task to describe the harmonic functions on the gasket . To that end in this section we also introduce a harmonic mapping of the Sierpinski gasket. This mapping allows us to give the geometric interpretation of the harmonic functions. The image of the Sierpinski gasket under this transformation is also a fractal; its geometrical construction is very simple, but involves two rescaling parameters. On that new fractal harmonic functions are simply linear functions, so we call the procedure harmonization of the fractal. After that the wellknown rescaling property of the energies on the pre-gaskets become a theorem from the elementary geometry. It should be pointed out that for the particular case of the Sierpinski gasket one can find the spectrum explicitly (see [3]) but in Sect. 2 an approach is presented which is applicable to all the objects of that nature. They are the so-called finitely ramified fractals. The difference is that instead of the natural resistor network and the explicit rescaling factor $5 / 3$ for the Sierpinski gasket one should admit connections with some conductivities between all the vertices of the elementary cell of such a gasket, provided selfsimilarity for the energy with a certain rescaling parameter $\varrho$. Generically those conductivities cannot be found explicitly, but the existence of this distribution of conductivities of the elementary cell of such a type of gasket was proved in [8]. We remark that the uniqueness of such a distribution of the conductivities is not established but that the number $\varrho$ is obviously uniquely defined thanks to uniform ellipticity arguments (this assertion is missing in [8]).

In Sect. 3 we consider the inhomogeneous pre-Sierpinski gasket. Now its bonds are equipped with two different conductivities: horizontal with $a$, others with $b$. In contrast with the previous case $(a=b)$ there is no reasonable pointwise (with respect to a set of admissible potentials) convergence of the pre-gasket energies. However, we relax the definition of the convergence and prove $\Gamma$-convergence of the energies, rescaled in the same manner as above. (See [11] for the general definition of $\Gamma$-convergence; in Sect. 3 it is given in our particular case.) We show that the $\Gamma$-limit is equal to $\sigma(a, b) \varepsilon$, where $\varepsilon$ is the standard energy, as in Sect. 2, and $\sigma(a, b)$ is some number. We call $\sigma(a, b)$ the effective conductivity of the inhomogeneous gasket, according to its physical sense. The main theorem includes explicit representation of $\sigma(a, b)$ in terms of the successive iterations of a given rational function. This representation shows that $\sigma(a, b)$ is invariant under a certain algebraic transformation, and enables us to investigate the analytic properties of the effective conductivity, as well as its asymptotics. We remark, for instance, that the set of $\sigma(a, b)$ essential singular points coincides with the Julia set [17] of that rational function mentioned above. That set is known to be the set on the line of some fractional Haussdorff dimension. It is reasonable to compare the structure of the singularities of $\sigma(a, b)$ with the analytic properties of the effective conductivity of two phase composites typical for homogenization theory geometry. In standard homogenisation theory the poles of such effective conductivity $\sigma(a, b)$, which are physically the electric resonances, are identical with the eigenvalues of the Bergman's spectral problem [14] (see [15] and also [16] for the explicit formulation 
of that problem). So in the case of the inhomogeneous Sierpinski gasket Bergman's problem has Kantor spectra. In standard homogenisation theory only discrete (e.g. dispersive periodic inclusions) and absolutely continuous (e.g. checkerboard structure) types of spectra were observed.

Another consequence of this invariance discussed above of $\sigma(a, b)$ under rational transformation is the logarithmic asymptotics of $\sigma(a, b)$ as $b / a \rightarrow \infty$, and both the gasket dimensions appear in that limit. We find in that limit

$$
\sigma(a, b) \sim a^{x} b^{1-x}, \quad x=\frac{\log \frac{6}{5}}{\log \frac{4}{3}}
$$

(compare with $x=1 / 2$ for the case of statistically equivalent phases in the homogenization theory).

We conclude the paper with a new proof of the Barlow-Bass [18] inequalities with the improved values of the constants concerning the limiting behavior of the preSierpinski carpet conductivity. This structure is obtained according to the following recurrent procedure: we take the unit square and paint it white, then we divide it into nine equal squares and paint the middle square black. We proceed making the same division and painting with all the rest eight white squares. Assume that the white set on the $n^{\text {th }}$ stage of this construction is filled with a conductor of conductivity one and the black set is dielectric. Then for the effective conductivity $C_{n}$ of this inhomogeneous structure for any $n$ the estimate holds true:

$$
c^{-1} \varrho^{n}<C_{n}<c \varrho^{n}
$$

for some fixed $\varrho, 0<\varrho<1$ with $c=4$ (see [18]). We remark that the explicit value of $C_{1}$ was found in [19]. Here it will be shown that those inequalities are true for $c=3 / 2$. This improvement was mainly achieved thanks to the application of $2 D$ interchange duality, and to the introduction of the relevant auxiliary networks, which better take into account inhomogeneity of this structure and in particular the singularities of the solution at the corners of each black square. The proof cannot be immediately generalized to the $3 D$ Sierpinski carpet and we do not know if the constant $3 / 2$ is optimal or not.

\section{Laplacian and Spectral Dimension of Gaskets}

Here we explain how to construct the Laplacian and define the spectral dimension of the Sierpinski gasket.

Let us first recall the definition of the Sierpinski gasket. Pose $a_{1}=(0,0)$, $a_{2}=(1,0)$, and $a_{3}=(1 / 2, \sqrt{3 / 2})$, let $V_{0}=\left\{a_{0}, a_{1}, a_{2}\right\}$ be the vertices of an equilateral triangle of side one. Define inductively

$$
V_{n+1}=V_{n} U\left(2^{-n} a_{2}+V_{n}\right) U\left(2^{-n} a_{3}+V_{n}\right) .
$$

(Here, and throughout the paper we use the notation $x+A=\{x+y, y \in A\}$ ). Now let

$$
V_{*}=\bigcup_{n=0}^{\infty} V_{n}
$$

and $V=\bar{V}_{*}$ (closure of $V_{*}$ ) be the Sierpinski gasket. 
Let $\mu_{n}$ denote the measure which assigns mass $3^{-n}$ to each point in $V_{n}$. It is wellknown that $\left\{\mu_{n}\right\}$ converges in vague topology to the Hausdorff $x^{d_{f}}$-measure $\mu$ on $V$ and $d_{f}=\log 3 / \log 2$. Thus $V$ has Hausdorff dimension $d_{f}$ and $\mu\left(T_{n}\right)=3^{-n}$ for any equilateral triangle $T_{n}$ whose vertices are one of the possible translations of $2^{-n} V_{0}$.

Let's assign conductivity one to each nearest neighbor bond in $V_{m}$, and consider pre-Sierpinski gasket as the resistor network. Then for the Joule heat we have the expression

$$
\varepsilon_{m}^{0}(f)=\sum_{\substack{x, y \in V_{m} \\|x-y|=2^{-m}}}(f(x)-f(y))^{2},
$$

where $f: V_{*} \rightarrow R$ is any function (given the potential of the vertices $V_{m}$ ). Suppose we fix potentials only on $V_{0}$, then the energy is

$$
\bar{\varepsilon}_{m}^{0}(\varphi)=\inf \left\{\varepsilon_{m}^{0}(f): f: V_{m} \rightarrow R,\left.f\right|_{V_{0}}=\varphi\right\}, \quad \varphi=\left(\varphi_{1}, \varphi_{2}, \varphi_{3}\right) .
$$

Simple calculation based on the symmetries and the similarity shows that

$$
\vec{\varepsilon}_{m}^{0}(\varphi)=\left(\frac{3}{5}\right)^{m}\left(\left(\varphi_{1}-\varphi_{2}\right)^{2}+\left(\varphi_{2}-\varphi_{3}\right)^{2}+\left(\varphi_{1}-\varphi_{3}\right)^{2}\right) .
$$

Indeed, thanks to $120^{\circ}$-rotation symmetry and obvious independence of the energy on the simultaneous shift of the potentials to the same constant, the energy is proportional to the energy of the initial triangle with unit conductivities, which is given by the expression (2.1) for $m=1$. We call that factor of proportionality the effective conductivity of the standard pre-Sierpinski gasket. By induction it suffices to prove (2.3) for $m=1$. In that case we consider antisymmetric distribution of the potential: $(0,1,-1)$, which leads to one equation for one unknown variable on $V_{1}$. Solving this equation and computing the energy of $V_{1}$, we get (2.3). So this means that effective conductivity of the $V_{m}$-resistor network is equal to $(3 / 5)^{m}$, and we have to introduce the rescaled energy

$$
\varepsilon_{m}(f)=\left(\frac{5}{3}\right)^{m} \varepsilon_{m}^{0}(f)
$$

in order to have something finite in the limit.

Proposition 1. For any $f: V_{*} \rightarrow R$ the energy $\varepsilon_{n}(f)$ is nondecreasing on $n$.

Proof. Let's denote by $f_{m+1}^{0}$ harmonic continuation of $f_{m}=\left.f\right|_{V_{m}}$ to $V_{m+1}$, which can be defined as a solution of the variational problem

$$
\inf \left\{\varepsilon_{m+1}(g): g: V_{m+1} \rightarrow R,\left.g\right|_{V_{m}}=f_{m}\right\} .
$$

Then by definition of $f_{m+1}^{0}$ and rescaling property we get

$$
\varepsilon_{m+1}(f) \geq \varepsilon_{m+1}\left(f_{m}^{0}\right)=\varepsilon_{m}(f)
$$

as required.

We also need the following.

Proposition 2. For any $f: V_{*} \rightarrow R$,

$$
\sup _{x, y \in V_{*}} \frac{|f(x)-f(y)|}{|x-y|^{\alpha}} \leq 6 \sqrt{\sup _{n \geq 0} \varepsilon_{n}(f)},
$$

where $\alpha=(\log 5 / 3) / \log 4$. 
We skip the proof which is a bit long but follows directly from the definition of the energy (compare with the much more delicate result from [6]).

According to Proposition 1 we can introduce for given $f: V_{*} \rightarrow R$ the limiting energy as, probably infinite limit

$$
\varepsilon(f)=\lim _{n \rightarrow \infty} \varepsilon_{n}(f) .
$$

Pose now

$$
H^{1}=\left\{f: V_{*} \rightarrow R: \varepsilon(f)<\infty\right\} .
$$

Then thanks to Proposition 2 each $f \in H^{1}$ has continuation to $V$, which belongs to a standard Hölder space with the norm given by 1.h.s. of $(2.6)-C^{\alpha}(V)$. Let's define $C_{0}(V)=\left\{f \in C(V):\left.f\right|_{V_{0}}=0\right\}$, and introduce the norm

$$
\|f\|_{1}=(\varepsilon(f))^{1 / 2} .
$$

The functional space $C_{0}(V) \cap H^{1}$ is then a Hilbert space, and we denote it $\stackrel{\circ}{H}^{1}$. Obviously the space $L_{2} \equiv L_{2}(V, d \mu)$ contains $\stackrel{\circ}{H}$, which is dense in $L_{2}$ (see Lemma 1 below). In that case for given $v \in L_{2}$ we have the linear functional

$$
\langle v, u\rangle=\int_{v} v u d \mu
$$

which is continuous on $\stackrel{\circ}{H}^{1}$. Then the expression

$$
\|v\|_{-1}=\sup \left\{\langle v, u\rangle: u \in \stackrel{\circ}{H}^{1},\|u\|_{1}=1\right\}
$$

defines the pre-norm on $L_{2}$, and we denote by $H^{-1}$ the closure of $L_{2}$ with respect to this pre-norm. Now we have three Hilbert spaces $H^{-1}, L_{2}, \stackrel{\circ}{H^{1}}$ and the embeddings

$$
\stackrel{\circ}{H^{1}} \subset L_{2} \subset H^{-1} \text {. }
$$

Denote $\varepsilon(f, g)$ the scalar product of $f, g \in \stackrel{\circ}{H}^{1}$.

Then relation

$$
\langle\Delta f, g\rangle=\varepsilon(f, g)
$$

defines uniquely the isomorphism

$$
\Delta: \stackrel{\circ}{H}^{1} \approx H^{-1}
$$

and we call this operator the Laplacian. We also use the same name and notation for the unbounded operator $\Delta: L_{2} \rightarrow L_{2}$, which has the domain $D(\Delta)=\left\{f \in \stackrel{\circ}{H^{1}}: \Delta f \in\right.$ $\left.L_{2}\right\}$.

Remark that this way of introduction of the Laplacian permits us to prove in the usual manner, via the Hilbert space formulation the existence and uniqueness of the solution to the problem $\Delta u=0, u=\varphi$ on $V_{0}$. So here the role of the boundary is played by three points of the initial triangle, consequently the dimension of the space of the harmonic functions is equal to three. Thanks to Proposition 1 harmonic functions are of the class $C^{\alpha}$ and take limiting values in the classical sense. Explicit expression for the Laplacian of an arbitrary function is not known, but for a function 
$g_{n}$ which is harmonic inside each triangle $T_{n}$ the image of the Laplacian over $5^{n}$ is the function, which is identically zero on $V \backslash V_{n}$ and at the point $x \in V_{n}$ equals to the arithmetic mean of the nearest neighbors values minus $g_{n}(x)$.

Proposition 3. The operator $\Delta: L_{2} \rightarrow L_{2}$ is selfadjoint and has discrete spectra $\left\{\lambda_{k}\right\}$ $0<\lambda_{1} \leq \lambda_{2} \leq \ldots \leq \lambda_{k} \leq \ldots \rightarrow \infty$.

Proof. $\Delta$ is selfadjoint in $L_{2}$ by definition and the rest of the required statement follows immediately from Proposition 2, as far as imbedding $L^{2} \supset C_{\alpha}$ is compact for $\alpha \geq 0$.

Now we are ready to define the spectral dimension of the Sierpinski gasket. Denote

$$
N(\lambda)=\operatorname{card}\left\{\lambda_{k}: \lambda_{k} \leq \lambda\right\}
$$

the Weyl distribution function of the Laplacian's spectrum.

Lemma 1. There exist $C_{1}, C_{2}>0$ such that

$$
C_{1} \lambda^{d_{s} / 2} \leq N(\lambda) \leq C_{2} \lambda_{1}^{d_{s} / 2}
$$

for $\lambda>0 . \operatorname{In}(1.8) d_{s}=\log 9 / \log 5$ and is called spectral dimension.

Proof. In order to obtain estimate (2.8) below we get the upper bound of the pregasket energy. Let's denote by $H_{n}$ the subspace of $L_{2}$ whose elements are harmonic inside each triangle $T_{n}$ and equal to zero on $V_{0}$. Then by simple calculation of the number of the points in $V_{n} \operatorname{dim} H_{n}=3\left(3^{n}-1\right) / 2$ and

$$
\{0\}=H_{0} \subset H_{1} \subset \ldots \subset H_{n} \subset \ldots ; \overline{U H}_{n}=L_{2} .
$$

By definition of $L^{2}$ the set of the continuous functions is dense in $L^{2}$ and according to Proposition 1 each continuous function can be approximated uniformly on $V$ by the function from $H_{n}$ for sufficiently large $n$.

Now for any $f \in H_{n}$ we have

$$
\varepsilon(f)=\varepsilon_{n}(f)=\left(\frac{5}{3}\right)^{n} \sum_{\substack{x, y \in V_{n} \\|x-y|=2^{-n}}}(f(x)-f(y))^{2} \leq 16 \cdot 5^{n}\|f\|_{L_{2}}^{2} .
$$

Let us define the operator $\Delta_{1}$ in $L_{2}$ by its invariant subspaces $\left\{H_{n}\right\}$ and eigenvalues $16 \cdot 5^{n}$. (Each such eigenvalue is taken with the multiplicity equal to the dimension of the orthogonal complement to $H_{n}$ in $H_{n+1}$.) Estimate (2.9) now means $\Delta<\Delta^{1}$ in the operator sense, hence $N(\lambda) \geq N^{1}(\lambda)$, where $N^{1}(\lambda)$ is a $\Delta^{1}$ Weyl distribution function. Then (2.8) and the lower bound can be handled by explicit evaluation of the $\Delta^{1}$ spectrum distribution.

To get (2.8) upper bound, we consider the same sequence of spaces $\left\{H_{n}\right\}$ but in the Hilbert space $\stackrel{\circ}{H}^{1}$. Denote $H_{n}^{\perp}$ the orthogonal complement to $H_{n}$ in $\stackrel{\circ}{H}^{1}$. Then for any $f \in H_{n}^{\perp} \cap H_{n+1}$ we get by direct calculation $\left.f\right|_{V_{n}}=0$ thanks to the orthogonality to $H_{n}$ and

$$
\varepsilon_{n+1}(f) \geq 5^{n}\|f\|_{L_{2}}^{2} .
$$


Fig. 1

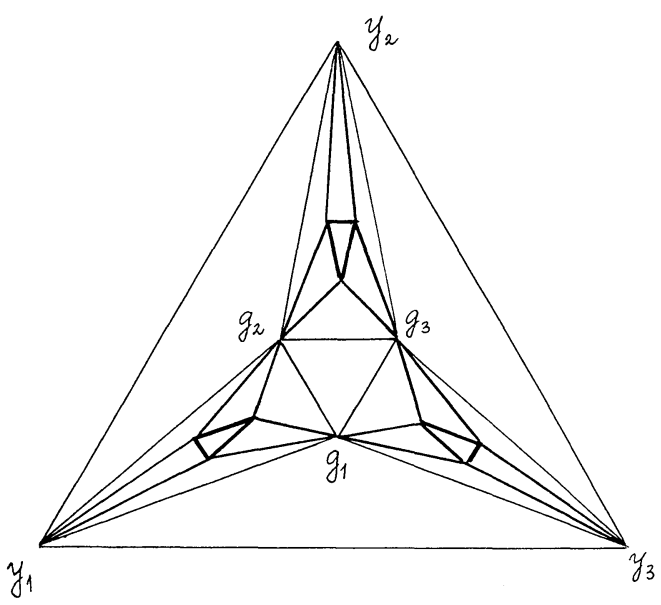

Henceforth, posing $f=\Delta^{-1 / 2} g$, from the last inequality we obtain

$$
\left(\Delta^{-1} g, g\right)_{L_{2}} \leq 5^{-n}\|g\|_{L_{2}}^{2} .
$$

Now we define operator $\Delta_{2}^{-1}$ in $L_{2}$ by its eigenspaces $\left\{\Delta^{1 / 2}\left(H_{n}^{\perp} \cap H_{n+1}\right)\right\}_{n=1}^{\infty}$ and eigenvalues $5^{-n}$. The previous estimate yields the operator inequality $\Delta>\Delta_{2}$. The upper bound (2.8) follows as above.

Now we are going to construct the harmonic functions on the Sierpinski gasket geometrically. There are three linearly independent harmonic functions on $V$ and one of them is identically constant. That means we can choose three vectors $y_{1}, y_{2}, y_{3} \in R^{2}$ such that triangle $G$ with vertices $\left(y_{1}, y_{2}, y_{3}\right)$ is nontrivial, and pose vector boundary conditions for the harmonic vector function $Z$

$$
\begin{gathered}
\Delta \mathbf{Z}=0, \quad \mathbf{Z}=\left(\mathbf{Z}_{1}, \mathbf{Z}_{2}\right) \in R^{2}, \\
\left.\mathbf{Z}\right|_{x=a_{i}}=y_{\imath},
\end{gathered}
$$

where $i=1,2,3$. Denote $y=\left(y_{1}, y_{2}, y_{3}\right)$, and introduce the new triple of $R^{2}$-vectors $\left(g_{1}(y), g_{2}(y), g_{3}(y)\right)$ as follows $g(y)=\left(g_{1}(y), g_{2}(y), g_{3}(y)\right)$ :

$$
g(y)=\frac{1}{5}\left(2\left(y_{1}+y_{2}\right)+y_{3}, 2\left(y_{1}+y_{3}\right)+y_{2}, 2\left(y_{2}+y_{3}\right)+y_{1}\right) .
$$

That new triple has the sense of the values of $\mathbf{Z}$ on $V_{1} \backslash V_{0}$, and that is easy to check solving the equations for $Z$. Let us define three triangles $G_{i}, i=1,2,3$ with the vertices $\left(y_{1}, g_{1}(y), g_{2}(y)\right),\left(y_{2}, g_{2}(y), g_{3}(y)\right),\left(y_{3}, g_{3}(y), g_{1}(y)\right)$ respectively (see Fig. 1$)$. Now we describe the map $g$ geometrically. Starting from the triangle $T_{y}\left(y_{1}, y_{2}, y_{3}\right)$ triangle $T_{g}\left(g_{1}(y), g_{2}(y), g_{3}(y)\right)$ can be found via the following simple procedure. We divide each side of $T_{y}$ onto five equal segments, then join the respective points of the adjacent sides and find $T_{g}$ as the central of that triangulation (see Fig. 2). Energy of the potential distribution $Z$, which dissipates on the bond of the initial pre-gasket now is nothing else but the squared length of the bond of the new pre-gasket obtained via transformation $g$. So the energy rescaling property now means the following.

Theorem 1. Summing up squared sides of arbitrary triangle $G$ we get five thirds times the sum of squared sides of all the triangles $G_{1}, G_{2}, G_{4}$.

Geometrically map $g$ is shown on Fig. 1. We can proceed in the same manner applying the map $g$ to each of the triangles $G_{1}, G_{2}, G_{3}$ and this is also shown on 
Fig. 2

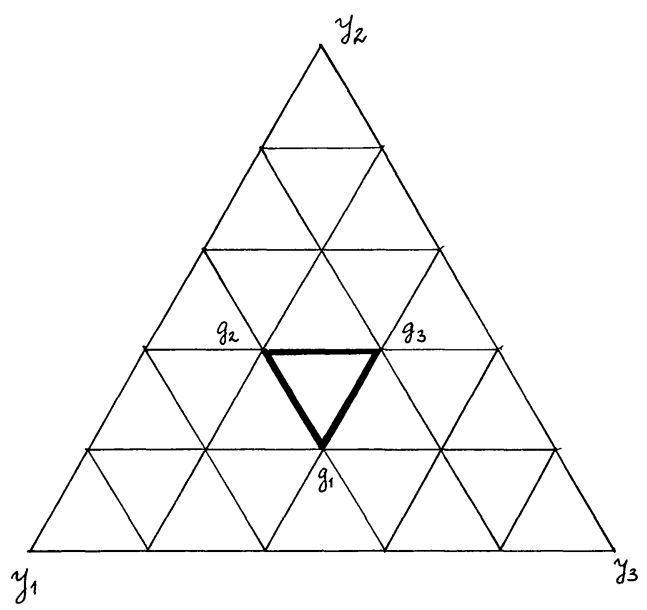

Fig. 1. That gives the values of $\mathrm{Z}$ on $V_{2} \backslash V_{1}$ and so on. Doing this way we obtain the map $\hat{g}: V_{*} \rightarrow G$. One can easily check that the continuation of $g$ to all $V$ gives a homeomorphism $\hat{g}: V \approx G V$, where $G V$ is the closure of $\hat{g} V_{*}$ in $G$.

Remark 1. We can induce the energy, the Laplacian, and the Hausdorff measure from $V$. Then by definition linear functions will be harmonic and the spectral dimension is equal to $d_{s}$. Unfortunately, we are not able to calculate the Hausdorff dimension of $G V$, but covering $\hat{g} V_{m}$ primitive triangles by $3^{n}$ balls of diameter $5^{-n}$ we obtain the estimate $d_{H}(G V) \leq d_{s}$. The natural hypothesis is: $d_{H}(G V)=d_{s}$. Geometric construction of $G V$ presented above involves obviously two rescaling parameters $1 / 5,3 / 5$ and that yields to the difficulties in the $G V$ Hausdorff dimension evaluation.

Remark 2. With the help of harmonic coordinates we can describe explicitly the dense set in $H^{1}$ that is:

$$
\left\{f(\mathbf{Z}): f \in C^{1}(G)\right\}
$$

Remark. The same results as here hold true for other nested fractals as far as the rescaling property of the energy is fulfilled. Existence of the unique rescaling parameter follows from [8] and standard ellipticity arguments, but one should consider a more general type of energy (not only nearest neighbors are connected).

With respect to these observations it is natural to call any gasket on which linear functions are harmonic, harmonic gasket, and call $G V$ harmonization of the gasket $V$. The next hypothesis is that the Laplacian on the harmonic gaskets is a second order differential operator with the usual partial derivatives but with coefficients of very special and irregular type.

\section{Homogenization on the Sierpinski Gasket}

Here we consider inhomogeneous pre-gasket, which sides have two different conductivities. Let's denote by $l_{1}, l_{2}, l_{3}$ the sides of the triangle $T_{0}$, joining the vertices 
$\left\{a_{1}, a_{2}\right\},\left\{a_{2}, a_{3}\right\},\left\{a_{3}, a_{1}\right\}$ respectively. For given $a, b>0$ we introduce local conductivity

$$
\sigma(e)=\left\{\begin{array}{l}
a, e\left\|l_{1} \quad e\right\| l_{2} \\
b, e \| l_{3}
\end{array}\right.
$$

(here $\|$ means being parallel) and $e$ is any side of any triangle $T_{n}$. Let's define the energy as

$$
\varepsilon_{n}(a, b ; f)=\left(\frac{5}{3}\right)^{n} \sum_{\substack{x, y \in V_{n} \\|x-y|=2^{-n}}} \sigma(x-y)(f(x)-f(y))^{2}
$$

where $f \in H^{1}$.

We need an important notion of $\Gamma$-convergence (or equivalent epi-convergence) from nonlinear analysis (see [11]). Let us recall it in the current situation. Sequence $\left\{\varepsilon_{n}\right\}_{n=1}^{\infty}$ of the functionals on $H_{1}$ is said to be $\Gamma$-convergent on $H_{1}$ to a functional $\tilde{\varepsilon}$ (notion $\Gamma-\lim \varepsilon_{n}=\bar{\varepsilon}$ ) iff

(i) for any weakly in $H^{1}$ convergent sequence $f_{n} \rightarrow f$ we have

$$
\liminf _{n \rightarrow \infty} \varepsilon_{n}\left(f_{n}\right) \geq \tilde{\varepsilon}(f)
$$

(ii) there exists a sequence $\left\{f_{n}^{0}\right\}_{n=1}^{\infty}, f_{n}^{0} \in H^{1}$ such that $f_{n}^{0} \rightarrow f$ in $H^{1}$ weakly and

$$
\lim _{n \rightarrow \infty} \varepsilon_{n}\left(f_{n}^{0}\right)=\tilde{\varepsilon}(f) .
$$

We remark that the sequence of the energies $\left\{\varepsilon_{n}\right\}$ from Sect. $2 \Gamma$-converges to $\varepsilon=\|\cdot\|_{1}^{2}$ which follows from the definition, but generically $G$-convergence is weaker than the pointwise convergence which we have in this example.

Now let's discuss the inhomogeneous case.

Theorem 2. For any $a, b>0$ sequence $\left\{\varepsilon_{n}(a, b ; \cdot)\right\}_{n=1}^{\infty}$ defined in (3.1), (3.2) $\Gamma$ converges

$$
\Gamma-\lim \varepsilon_{n}(a, b ; \cdot)=\hat{\sigma}(a, t)\|\cdot\|_{1}^{2} ; t=b a
$$

and for the number $\hat{\sigma}(a, t)$ we have the representation

$$
\hat{\sigma}(a, t)=a \prod_{k=0}^{\infty} \frac{5+10 R_{k}(t)}{6+9 R_{k}(t)}
$$

where

$$
R_{0}(t)=t, R_{1}(t)=\frac{3 t^{2}+6 t+1}{4 t+6}, \ldots, R_{k}=R_{1}\left(R_{k-1}^{\prime}(t)\right), \ldots
$$

and the product in (3.3) converges for any $t>0$. 
We call $\hat{\sigma}(a, t)$ the effective conductivity of the inhomogeneous Sierpinski gasket. It is interesting to remark that the obvious anisotropy of the inhomogeneous resistor network disappears in the limit, since all the process of conductivity could be characterized by one number-conductivity of the side of one triangle. To explain this at-first-sight strange fact we can mention that nevertheless it is reasonable, because for any path going in some direction we can find a path going in another prescribed direction such that the density of $a^{-s}$ and $b^{-\frac{-s}{}}$ on those paths is asymptotically the same. Let us pose $\hat{\sigma}(t)=\hat{\sigma}(1, t), R=R_{1}$ for the sake of shortness and establish several properties of $\hat{\sigma}(t)$. The first follows directly from (3.3).

Corollary 1. The function $\hat{\sigma}(t)$ has the extension which is analytic away from $([-\infty,-3 / 2])$ and satisfies the relation

$$
\hat{\sigma}(R(t))=\frac{6+9 t}{5+10 t} \hat{\sigma}(t) .
$$

Remark 4. From the representation (3.3) one can easily deduce also the structure of singularities of $\hat{\sigma}$. In fact $\hat{\sigma}$ has a countable set of poles which are the zeros of denominators in the representation (3.3) and consequently is the set of all $R$ predecessors of $-2 / 3$. All limiting points of that set form the set of essential singularities of $\hat{\sigma}$. The last is the Julia set of the rational function $R$ (see [17]). In our case the Julia set coincides with the closure of all the predecessors of -1-unique repulsive fixed point of $R$.

Corollary 2. For $\hat{\sigma}(t)$ takes place the limiting relation

$$
\lim _{t \rightarrow \infty} \frac{\log \hat{\sigma}(t)}{\log t}=\frac{\log \frac{10}{9}}{\log \frac{4}{3}} .
$$

Later on we show that (3.6) is a consequence of the invariance property (3.5).

Let us start now with the proof of Theorem 2. First we investigate transformation of conductivities:

$$
\varepsilon_{n}^{\varphi}(a, b)=\inf \left\{\varepsilon_{n}(a, b ; f): f \in H^{1},\left.f\right|_{\partial V}=\varphi\right\} .
$$

Taking into account that the isosceles property is preserved thanks to the symmetry of the problem with respect to the median, and this means simply that we can consider $\varepsilon_{n}^{\varphi}(a, b)$ as the energy $\varepsilon_{0}^{\varphi}\left(a_{n}, b_{n}\right)$ for some $a_{n}, b_{n}$, by a long, but direct calculation, we get the following.

Proposition 4. For any $n \geq 0$,

$$
\varepsilon_{n+1}^{\varphi}(a, b)=\varepsilon_{n}^{\varphi}\left(a_{1}, b_{1}\right)
$$

where

$$
a_{1}=a \frac{a+2 b}{2 a+3 b}, \quad b_{1}=\frac{(a+2 b)\left(a^{2}+3 b^{2}+6 a b\right)}{2(3 a+2 b)(2 a+3 b)}
$$

In variables $a, t=b / a$ transformations (3.9) mean

$$
a_{1}=a \frac{1+2 t}{2+3 t} \quad t_{1}=\frac{3 t^{2}+6 t+1}{4 t+6} .
$$


Proposition 5. There exists the limit

$$
\lim _{n \rightarrow \infty} \varepsilon_{n}^{\varphi}(a, b)=a \prod_{k=1}^{\infty} \frac{5+10 R_{k}(t)}{6+9 R_{k}(t)} \varepsilon_{0}(\varphi),
$$

where $R_{k}$ is defined as in (3.4) and $\varepsilon_{0}(\varphi)$ in (1.1). The product in (3.11) converges for any $t>0$.

Proof. Rational mapping $t \rightarrow R(t)$ has only one attractive fixed point $t=1$, hence analyzing the graph of $R(t)$ we see that for any $t>0\left|R_{K}(t)-1\right|<C_{t} e^{-\beta K}$ with some $C_{t}>0, \beta>0$ where $C_{t}$ probably depends on $t$. Then for any fixed $t>0$ the infinite product in (3.11) converges, and it remains to remark that its $n_{\text {th }}$ partial product coincides with $\varepsilon_{n}^{\varphi}(a, b)$ according to (3.10).

To prove the theorem, now, it suffices to establish $\Gamma$-convergence of $\varepsilon_{n}(a, b ; f)$ to $\hat{\sigma}(a, t) \varepsilon(f)$. Let's verify the first requirement of the definition. Assume we have a sequence $\left\{f_{n}\right\}$ and $f_{n} \rightarrow f$ in $H^{1}$ weakly. We denote by $f_{n}$ the function $\tilde{f}_{n}: V_{2 n} \rightarrow R$ which minimizes the problem

$$
\inf \left\{\varepsilon_{2 n}(a, b ; g): g-\left.f_{n}\right|_{V_{n}}=0\right\} .
$$

Then according to this definition we have

$$
\varepsilon_{2 n}\left(a, b ; f_{2 n}\right) \geq \varepsilon_{2 n}\left(a, b ; \tilde{f}_{n}\right)=\varepsilon_{n}\left(a_{n}, b_{n} ; f_{n}\right),
$$

where $a_{n}, b_{n}$ are the $n^{\text {th }}$ iterations of (3.9). Proposition 5 yields for $f_{n}^{0}=\left.f_{n}\right|_{V_{n}}$,

$$
\varepsilon_{n}\left(a_{n}, b_{n} ; f_{n}\right) \geq\left(\tilde{\sigma}(a, t)-\delta_{n}\right) \varepsilon\left(f_{n}^{0}\right),
$$

where $\delta_{n} \rightarrow 0, n \rightarrow \infty$. Let's denote by $\tilde{f}_{n}^{0}$ harmonic inside each $T_{n}$-triangle continuation of $f_{n}^{\circ}$ onto $V$. Then we have $\tilde{f}_{n}^{0} \rightarrow f$, in $H^{1}$ weakly since $\max _{V}\left|\tilde{f}_{n}^{0}-f\right| \rightarrow$ 0 as $n \rightarrow \infty$ and the sequence $\left\{\tilde{f}_{n}^{0}\right\}$ is bounded in $H^{1}$. Hence, we get

$$
\lim _{n \rightarrow \infty} \varepsilon\left(f_{n}^{0}\right) \equiv \lim _{n \rightarrow \infty} \varepsilon\left(f_{m}^{0}\right) \geq \varepsilon(f)
$$

thanks to the nondecreasing of the norm of weakly convergent sequence. Finally we get the desired property

$$
\lim _{n \rightarrow \infty} \inf \varepsilon_{n}\left(a, b ; f_{n}\right) \geq \hat{\sigma}(a, t) \varepsilon(f)
$$

for an arbitrary weakly convergent sequence $\left\{f_{n}\right\}$.

Now to verify (ii) we construct the sequence $\left\{f_{n}^{0}\right\}$ in the following way. We assign $f_{n}$ to be the minimizant of the variational problem

$$
\inf \left\{\varepsilon_{2 n}(a, b ; g): g-\left.f\right|_{V_{n}}=0\right\}
$$

and continue it harmonically inside each $T_{n}$-triangle. Then, as above, $f_{n} \rightarrow f$ in $H^{1}$ when $n \rightarrow \infty$, and

$$
\varepsilon_{2 n}\left(a, b ; f_{n}\right)=\varepsilon_{n}\left(a_{n}, b_{n} ; f\right)=\hat{\sigma}(a, t)\left(1+\delta_{n}\right) \varepsilon_{n}(f),
$$

where again $\delta_{n} \rightarrow 0, n \rightarrow \infty$. So in view of (1.7) we have

$$
\Gamma-\lim \varepsilon_{n}(a, b ; \cdot)=\hat{\sigma}(a, t) \varepsilon(\cdot)
$$

as desired. The theorem is proved. 
Let us prove Corollary 2. To that end, for any $\varepsilon>0$ we shall introduce two functions $\sigma_{ \pm}^{\varepsilon}$ such that for sufficiently large $t$,

$$
c_{1}^{\varepsilon} t^{\alpha-\varepsilon} \leq \sigma_{-}^{\varepsilon} \leq \hat{\sigma}(t) \leq \sigma_{+}^{\varepsilon} \leq c_{2}^{\varepsilon} t^{\alpha+\varepsilon},
$$

where $\alpha=(\log 10 / 9) /(\log 4 / 3)$. In order to construct $\sigma_{-}^{\varepsilon}$ we choose $T_{\varepsilon}$ so large that $R(t)<\left(\frac{3}{4}+\varepsilon^{-}\right) t$ for $t>T_{\varepsilon}$. Pose

$$
\sigma_{-}^{1}=\inf \left\{\hat{\sigma}(t): T_{\varepsilon} \leq t \leq\left(\frac{4}{3}+\varepsilon\right) T_{\varepsilon}\right\}=\hat{\sigma}\left(T_{\varepsilon}\right)
$$

and define $\sigma_{-}^{\varepsilon}(t)$ as a stepwise function

$$
\sigma_{-}^{\varepsilon}(t)=\sigma_{-}^{1}\left(\frac{10}{9}\right)^{k} \quad \text { for } \quad t: T_{\varepsilon}\left(\frac{4}{3}+\varepsilon\right)^{k} \leq t \leq\left(\frac{4}{3}+\varepsilon\right)^{k+1} T_{\varepsilon},
$$

$k=0,1, \ldots$ Then the lower bound (3.16) follows from Corollary 1 and estimate $(5+10 t) /(6+9 t)>10 / 9$. Similarly, setting

$$
\sigma_{+}^{1}=\sup \left\{\hat{\sigma}(t): \tau_{\varepsilon}<t<\left(\frac{4}{3}-\varepsilon\right) \tau_{\varepsilon}\right\}=\hat{\sigma}\left(\left(\frac{4}{3}-\varepsilon\right) \tau_{\varepsilon}\right),
$$

where $\tau_{\varepsilon}$ is chosen such that

$$
\frac{5+10 t}{6+9 t}<\frac{10}{9}+\varepsilon \text { for } t>\tau_{\varepsilon} .
$$

We introduce

$$
\sigma_{+}^{\varepsilon}(t)=\left(\frac{10}{9}+\varepsilon\right)^{k} \quad \text { for } \quad t: \tau_{\varepsilon}\left(\frac{4}{3}\right)^{k} \leq t<\left(\frac{4}{3}\right)^{k+1} \tau_{\varepsilon},
$$

and taking into account the obvious estimate $R(t)>\frac{3}{4} t$ we finish with the upper bound in (3.16).

\section{Effective Conductivity of the Sierpinski Carpet}

Let's recall the construction of the Sierpinski carpet. We denote by $F_{0}=[0,1]^{2}$ the unit square; divide it into nine equal squares, and paint the central black, others white. So $F_{1}=F_{0} \backslash(1 / 3,2 / 3)^{2}$ is the white set. Then we repeat the same operation for each of remaining white squares, painting the central squares of each of them black and keeping the rest white. Proceeding this way we denote by $F_{n}$ the white set on the $n^{\text {th }}$ stage of this construction. The set $F_{n}$ consists then of $8^{n}$ white squares of side $3^{-n}$.

Now we introduce local conductivity

$$
a_{n}(x ; \delta)=\left\{\begin{array}{ll}
1, & x \in F_{n} \\
\delta, & x \in F_{0} \backslash F_{n}
\end{array},\right.
$$

where $0 \leq \delta \leq \infty$. The effective conductivity of this structure can be defined as follows:

$$
C_{n}(\delta)=\inf \left\{\int_{F_{0}} a_{n}(x ; \delta)|\nabla u|^{2} d x: u\left(0, x_{2}\right)=0, u\left(1, x_{2}\right)=1, u \in H^{1}\left(F_{0}\right)\right\}
$$

where $H^{1}\left(F_{0}\right)$ is a Sobolev space of functions, having square integrable gradients. The well known interchange equality [10] (for general setup see [13]) says that

$$
C_{n}(\delta)=C_{n}^{-1}\left(\delta^{-1}\right)
$$




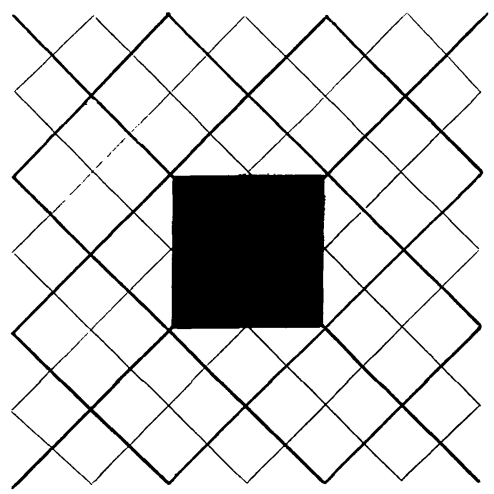

Fig. 3 . $-L_{1}^{*}$ $-L_{1}^{0}$

We use $0<\delta<\infty$ in order to have the solution of (4.1) to be defined all over $F_{0}$, for such $\delta$ the solution of (4.1) is of the class $C^{\beta}\left(F_{0}\right)$, where the Hölder exponent $\beta$ is strictly positive. The effective conductivity is continuous function with respect to $0 \leq \delta \leq \infty$ (see for example [11]) and for the sake of shortness we pose $C_{n}=C_{n}(0)$.

Theorem 1. There exists $\varrho$ such that

$$
2 / 3 \varrho^{n} \leq C_{n} \leq 3 / 2 \varrho^{n}
$$

To prove this theorem it suffices to establish the inequalities

$$
2 / 3 C_{n} C_{m} \leq C_{n+m} \leq 3 / 2 C_{n} C_{m}
$$

(see [20] problem 99). In order to do that we denote by $Z_{1}^{n}$ the solution of (4.1) and by $Z_{2}^{n}\left(x_{1}, x_{2}\right)=Z_{1}^{n}\left(x_{2}, x_{1}\right)$ assuming $0 \leq \delta \leq \infty$. Pose $Z^{n}=\left(Z_{1}^{n}, Z_{2}^{n}\right)$, then transformation $Z^{n}: F_{0} \rightarrow F_{0}$ has two basic properties:

(i) $\mathrm{Z}$ preserves the sides and the diagonals of the square $F_{0}$.

$$
\begin{aligned}
& \int_{F_{0}} a_{n}(x ; \delta) \nabla \mathbf{Z}_{i}^{n} \nabla \mathbf{Z}_{j}^{n} d x=4 \int_{T_{k}} a_{n}(x ; \delta) \nabla \mathbf{Z}_{i}^{n} \nabla \mathbf{Z}_{j}^{n} d x=C_{n} \delta_{\imath j} ; \\
& \quad i, j=1,2 ; k=1, \ldots, 4
\end{aligned}
$$

where $T_{k}$ is one of the four triangles bounded by the sides and the diagonals of the square $F_{0}$, and $\delta_{i j}$ is Kroneckers delta. Now we make standard triangulation of $\mathrm{F}_{0}$ with the vertices at the lattice $3^{-m} Z^{2}$ drawing the diagonals inside elementary square of side $3^{-m}$. Let's denote the vertices of this triangulation (i.e. $F_{0} \cap 3^{-m} Z^{2}$ and centers of elementary squares) by $L_{m}$ (see Fig. 3). Then for any nesh function $\varphi: L_{m} \rightarrow R$ we can construct a piecewise linear continuous function $f_{\varphi}^{*} \in H^{1},\left.f_{\varphi}^{*}\right|_{L}=\varphi$, which is inside each triangle a linear combination of three functions $\left\{1, x_{1}, x_{2}\right\}$. But, as it was pointed out in [18] the remarkable property (i) enables us to do the same with three basis functions $\left\{1, \mathrm{Z}_{1}^{n}, \mathrm{Z}_{2}^{n}\right\}$. Doing this way we get continuous function $f_{\varphi} \in H^{1}$ such that $\left.f_{\varphi}\right|_{L}=\varphi$. Let's pose

$$
\varepsilon_{m}^{*}(\varphi ; \delta)=\int_{F_{0}} a_{n}(x ; \delta)\left|\nabla f_{\varphi}^{*}\right|^{2} d x
$$


and

$$
C_{m}^{*}(\delta)=\inf \left\{\varepsilon_{m}(\varphi ; \delta): \varphi\left(0, x_{2}\right)=0, \varphi\left(1, x_{2}\right)=1\right\} .
$$

This number has the sense of an effective conductive of the respective inhomogeneous nesh with conductivities of white bonds equal to 1 and black to $\delta$ (a bond has the same colour as the cell). Denote this network by $L_{m}^{*}$. With the help of the solution $\phi$ of the problem (4.6) we construct the test function for the problem (4.1) of the order $n+m$. Assume the structure $F_{n}$ tesselates $Z^{2}$-periodically all the $R^{2}$, or in the other words the function $a_{n}(x, \delta)$ is continued to $R^{2} Z^{2}$-periodically. Then the vector-function $Z^{n}(x)$ could be considered as the function on $R^{2}$ such that $Z^{n}(x)-x$ is $Z^{2}$-periodic. Let us consider the rescaled function

$$
Z^{n, m}(x)=3^{-m} Z^{n}\left(3^{m} x\right) .
$$

According to (i) the map $\mathrm{Z}^{n, m}: R_{x}^{2} \rightarrow R_{z}^{2}$ preserves triangulation $\mathrm{L}_{m}^{*}$, i.e. the image of each triangle on the $R_{x}^{2}$-plane lies in the same triangle of the $R_{z}^{2}$-plane and the image of its boundary is the boundary. That means we can substitute to the piecewise linear functions $f_{\phi}^{*}(x)$ the mapping $Z^{n, m}(x)$ and introduce the test functions

$$
f_{\phi}(x)=f_{\phi}^{*}\left(Z^{n, m}(x)\right)
$$

which inside each triangle of $\mathrm{L}_{m}^{*}$ is the linear combination of $\left(1, Z_{1}^{m, n}(x), Z_{2}^{m, n}(x)\right)$ with the same coefficients as $f_{\phi}^{*}(x)$ is a combination of $\left(1, x_{1}, x_{2}\right)$. Then according to (ii) we have the relation

$$
\int_{T_{k, p}^{m}} a_{n}\left(3^{m} x ; \delta\right)\left|\nabla f_{\phi}(x)\right|^{2} d x=C_{n}(\delta) \int_{T_{k, p}^{m}}\left|\nabla f_{\phi}^{*}(x)\right|^{2} d x
$$

for any triangle $T_{k, p}^{m}$ of our triangulation. Pose now $F_{m}=\bigcup_{k} F_{k, m}$, where $k=\left(k_{1}, k_{2}\right)$ is such that $k+3^{-m} Z^{2} \in F_{0}, 0 \leq k_{1}, k_{2}<1$, and $F_{k, m}=k+3^{-m} F_{0}$,

$$
\begin{array}{cl}
a_{m}(k, \delta)=1 & \text { if } F_{k, m} \text { is white }, \\
a_{m}(k, \delta)=\delta, & \text { if } F_{k, m} \text { is black } .
\end{array}
$$

Then by definition

$$
a_{n+m}\left(x ; \delta^{2}\right) \leq a_{n}\left(3^{m} x ; \delta\right) a_{m}(x ; \delta)
$$

for $\delta \leq 1$, hence assuming $\phi$ to be the solution of (4.6) we get, thanks to (ii), (4.7) and the construction of the function $g_{\phi}(x)$ :

$$
\begin{aligned}
C_{n+m}\left(\delta^{2}\right) & \leq \int_{F_{0}} a_{n}\left(3^{m} x ; \delta\right) a_{m}(x ; \delta)\left|\nabla f_{\phi}(x)\right|^{2} d x \\
& =\sum_{k} a_{m}(k, \delta) \int_{F_{k, m}} a_{n}\left(3^{m} x ; \delta\right)\left|\nabla f_{\phi}(x)\right|^{2} d x \\
& =C_{n}(\delta) \int_{F_{0}} a_{m}(x ; \delta)\left|\nabla f_{\phi}^{*}(x)\right|^{2} d x=C_{n}(\delta) C_{m}^{*}(\delta) .
\end{aligned}
$$

The same arguments and the relation

$$
a_{n+m}(x ; \delta) \leq a_{n}\left(3^{m} x ; \delta\right) a_{m}(x, \delta)
$$


for $\delta \geq 1$ yields

$$
C_{n+m}(\delta) \leq C_{n}(\delta) C_{m}^{*}(\delta)
$$

Let us now use duality arguments (see $[10,13]$ ) in order to get from (4.9) the bound from below. To that end introduce the shifted network $L_{m}^{0}$ (see Fig. 3) which is obtained from $L_{m}^{*}$ as the union of all the perpendiculars to the centers to all the bonds of $\mathrm{L}_{m}^{*}$, and lies inside $F_{0}$. Assign conductivity $\delta$ to all the bonds of $\mathrm{L}_{m}^{0}$ lying in the black set and one otherwise. Denote by $C_{m}^{*}(\delta), C_{m}^{0}(\delta)$ the effective conductivities of the networks $L_{m}^{*}, L_{m}^{0}$ respectively, omitting the argument if it is equal to zero. The claim is that

$$
C_{m}^{*}(\infty) \leq 3 / 2 C_{m}^{0}(\infty)
$$

With (4.10) we can rewrite (4.9) as

$$
C_{n+m}(\infty) \leq 3 / 2 C_{n}(\infty) C_{m}^{0}(\infty)
$$

and by duality together with (4.9) we have

$$
2 / 3 C_{n} C_{m}^{*} \leq C_{n+m} \leq C_{n} C_{m}^{*}
$$

since $\mathrm{L}_{m}^{0}$ is dual to $\mathrm{L}_{m}^{*}$. Then the conductivities are inverse: $C_{m}^{0}(\infty)=\left(C_{m}^{*}\right)^{-1}$, $C_{k}(\infty)=\left(C_{k}\right)^{-1}$. As it was remarked above the Dirichlet and Neumann boundary conditions on the respective parts of the boundary are equivalent, thanks to the symmetry of the periodicity conditions of $\left(u-x_{1}\right)$ in (4.1). The effective conductivities are inverse (for the continuous problem see [10], and absolutely the same reasoning is applicable to the dual networks). In particular from (4.11) we have $C_{m}^{*} \leq 3 / 2 C_{m}$ and (4.4) follows.

Now let us prove (4.10). To that end introduce the third network

$$
\mathrm{L}_{m}^{+}=F_{0} \cap 3^{-m}\left\{(1 / 2,1 / 2)+Z^{2}\right\}
$$

with the standard bonds of $Z^{2}$-connectedness and consider each such bond as two conductivities in the series divided by the center of the bond, which we call demi-site in order to distinguish those points from the original sites of the $L_{m}$-centers of the elementary $-3^{-m} F_{0}$ squares. We assign conductivity two to each half bond if it lies in the white set and infinity if it is in the black set. Then we'll get an inequality

$$
C_{m}^{+}(\infty) \leq C_{m}^{0}(\infty)
$$

where $C_{m}^{+}(\infty)$ is the effective conductivity of $\mathrm{L}_{m}^{+}$which is understood in the same manner as the effective conductivity of $L_{m}^{*}$ with the same boundary conditions at the left and the right side of the initial square $F_{0}$. By the definition of the network $L_{m}^{+}$ the energy is a quadratic form defined on the nesh-functions on the set of all sites and demisites. To prove the desired inequality (4.12) it suffices to prescribe the test potential for the variational problem for the effective conductivity of $\mathrm{L}_{m}^{+}$to be equal at the center of each bond, i.e. at all the demi-sites except the boundary to the solution of the analogous variational problem for $L_{m}^{*}$ and define the test potential on the sites as the arithmetic mean of its values on the demi-sites - nearest neighbours 


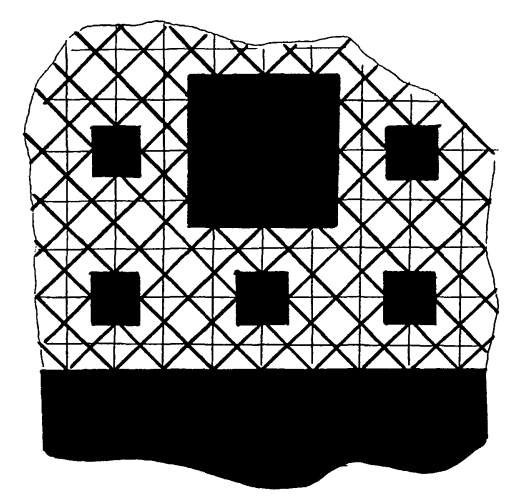

Fig. 4. $-L_{m}$

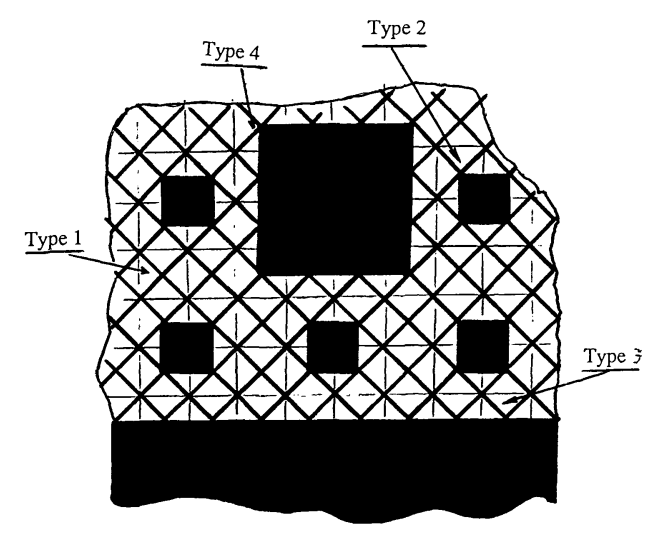

Fig. 5. $-L_{m}^{*}$ $-L_{m}^{+}$

(see Fig. 4). Now to get the inequality (4.12) explicitly, denote by $E_{+}(f)$ the energy for the network $L_{m}^{+}$of the arbitrary function $f$ given on the set of sites and demi-sites. Then we have because of the restriction

$$
\begin{aligned}
& C_{m}^{+}=\inf E_{+} \leq \inf \left\{E_{+}(f): f-\right.\text { such that its value at each site } \\
& \text { is equal to the arithmetic mean of values } \\
&\text { at the four neighbouring demi-sites }\} .
\end{aligned}
$$

Let us compute the value of $E_{+}$with the restriction above at the r.h.s. for the solution of the $L_{m}^{0}$-conductivity problem, using for each cell of $L_{m}^{0}$ containing a vertex of $L_{m}^{+}$ inequality

$$
\left(f_{1}-f_{2}\right)^{2}+\left(f_{2}-f_{3}\right)^{2}+\left(f_{3}-f_{4}\right)^{2}+\left(f_{4}-f_{1}\right)^{2} \geq f_{1}^{2}+f_{2}^{2}+f_{3}^{2}+f_{4}^{2},
$$

which is true for $f_{1}+f_{2}+f_{3}+f_{4}=0$, where $f_{i}, i=1, \ldots, 4$ denotes the values of the potential on the vertices of some cell of $L_{m}^{0}$ and the 1.h.s. exhibits the compound of the energy of $L_{m}^{0}$ while the r.h.s. is for $L_{m}^{+}$energy item. From here we get (4.12).

Now let us compare the energies of the resistor networks $L_{m}^{*}, L_{m}^{+}$introduced above, which we will compute assuming the distribution of the potential to be equal to the solution of the $L_{m}^{+}$-effective conductivity problem, filling in the missing $L_{m}^{*}$ values by arithmetic mean of four nearest neighbours. According to Fig. 5 we must 


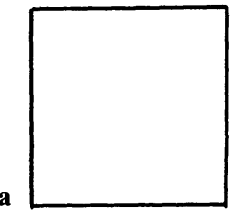

Fig. 6a, b

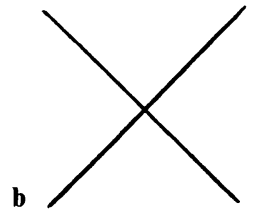

b

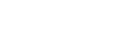

a

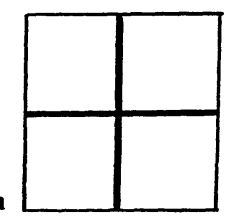

Fig. 7a, b.

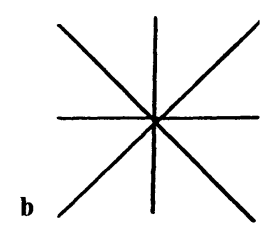

conductivity 2 ; - conductivity 1

distinguish four types of the cells of $L_{m}^{+}$. Look for the equivalent images presented at the Figs. 6-9a), b) for $L_{m}^{+}, L_{m}^{*}$ respectively. In each of those cases we seek for the maximum $\lambda$ such that

$$
\mathrm{E}^{+}(f) \geq \lambda \mathrm{E}^{*}(f)
$$

where $E^{+}, E^{*}$ are the energies of the considered parts of $L_{m}^{+}, L_{m}^{*}$ respectively computed on the same potential function $f$. In other words $\lambda$ is the minimum eigenvalue of $\mathrm{E}^{+}$when $\mathrm{E}^{*}$ defines the scalar product. We remark also that due to $\delta=\infty$ the potential is the same at the vertices connected with the black bond, so in the gometric representation we can reduce them to one point.

1) White cells, that means cells of $L_{m}^{+}$which are entirely in the white set (see Fig. 6). For the energies of such cells we have

$$
\begin{aligned}
\mathrm{E}^{+, 1}(f) & =\sum_{i=1}^{3}\left(f_{i+1}-f_{i}\right)^{2}, \\
\mathrm{E}^{*, 1}(f) & =\sum_{i=1}^{4}\left(f_{i}-f_{5}\right)^{2}, \\
f_{5} & =\left(\sum_{i=1}^{4} f_{i}\right) / 4
\end{aligned}
$$

respectively. Then

$$
\mathrm{E}^{+, 1}(f) \geq 2 \mathrm{E}^{*, 1}(f)
$$

2) Ensembles of four cells around the black square of the side $3^{-m}$ (see Fig. 7). We consider those cells together, so we must compare two quadratic forms of nine variables. But we can restrict the value at the center to be equal to the mean of its nearest neighbours in $\mathrm{L}_{m}^{+}$, which is certainly true for the solution of the $\mathrm{L}_{m}^{+}$-effective conductivity problem. To find the desired eigenvalue $\lambda$ remark that the forms and the restriction for the ninth variable under consideration are invariant with respect to $90^{\circ}$ rotation. This means that the respective eigenfunction has the same type of symmetry, hence we can assume $f_{2 i}=a, i=1, \ldots, 4 ; f_{2 i+1}=b, i=0, \ldots, 3$, and for the energies of the conglomerates of those cells which are

$$
\begin{aligned}
& \mathrm{E}^{+, 2}(f) \sum_{i=1}^{7}\left(f_{i+1}-f_{i}\right)^{2}+2 \sum_{i=1}^{4}\left(f_{2 i}-f_{9}\right)^{2} \\
& \mathrm{E}^{*, 2}(f)=\sum_{i=0}^{3}\left(f_{2 i+1}-f_{9}\right)^{2}+2 \sum_{i=1}^{4}\left(f_{2 \imath}-f_{9}\right)^{2},
\end{aligned}
$$




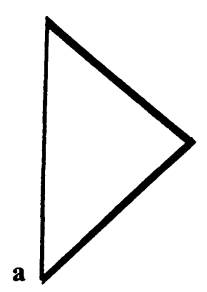

Fig. 8a, b.

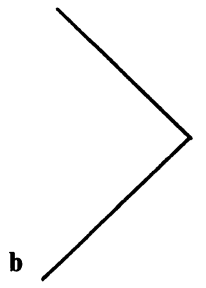
conductivity 2 ; - conductivity 1
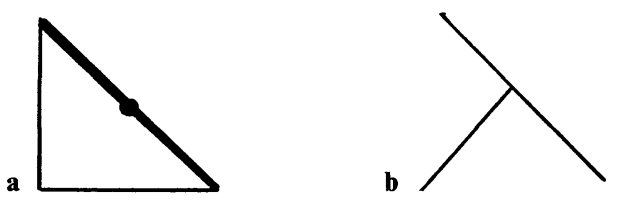

Fig. 9a, b. - conductivity 2 ;

- conductivity 1

we have then the inequality:

$$
\mathrm{E}^{+, 2}(f) \geq 2 \mathrm{E}^{*, 2}(f) .
$$

3) Cells which are half black (see Fig. 8). Here

$$
\begin{aligned}
& \mathrm{E}^{+, 3}(f)=\left(f_{1}-f_{2}\right)^{2}+2\left\{\left(f_{2}-f_{3}\right)^{2}+\left(f_{3}-f_{1}\right)^{2}\right\}, \\
& \mathrm{E}^{*, 3}(f)=\left(f_{2}-f_{3}\right)^{2}+\left(f_{1}-f_{3}\right)^{2},
\end{aligned}
$$

and we have the same inequality as in case 2 ).

4) Cells at the corners of the black squares of the side bigger than $3^{-m}$ (see Fig. 9). Here we have forms of three variables one of which is standard,

$$
\begin{aligned}
& \mathrm{E}^{+, 4}(f)=\left(f_{2}-f_{1}\right)^{2}+\left(f_{3}-f_{1}\right)^{2}+2\left(f_{2}^{2}+f_{2}^{2}\right), \\
& \mathrm{E}^{*, 4}(f)=f_{1}^{2}+f_{2}^{2}+f_{3}^{2},
\end{aligned}
$$

and we get

$$
\mathrm{E}^{+, 4}(f) \geq \mathrm{E}^{*, 4}(f) .
$$

Now to prove (4.10) we sum up the energies of all of those types, as far as each bond of $\mathrm{L}_{m}^{+}$takes part in two types of cells and never participates in two cells of the fourth type, we have computing the energy of the solution of the $L_{m}^{+}$-effective conductivity problem:

$$
3 / 2 C_{m}^{+}=3 / 2 \mathrm{E}_{m}^{+}(f) \geq \mathrm{E}_{m}^{*}(f) \geq C_{m}^{*}(\infty) .
$$

The theorem is proved.

Remark 5. Resume all the inequalities obtained above for $\delta=0$ :

$$
2 / 3 C_{m}^{*} \leq C_{m}^{0} \leq C_{m} \leq C_{m}^{*} \leq 3 / 2 C_{m}^{0} .
$$

Hence

$$
\begin{gathered}
2 / 3 \varrho^{m} \leq C_{m}^{*} \leq 9 / 4 \varrho^{m} \\
\left(2 / 3 C_{m}^{0}\right)^{1 / m} \leq \varrho \leq\left(3 / 2 C_{m}^{*}\right)^{1 / m}
\end{gathered}
$$

The last inequality can be usefull for numeric evaluation of $\varrho$.

We point out also that the lack of property (ii) for the network $L_{m}^{*}$ doesn't permit us to get subadditivity of $C_{m}^{*}$ the same way. From the proof we can see that the forms $E^{+}, E^{*}$ differ mostly at the corner points of type 4 ) and those points limit further progress in the evaluation of $C_{m}$ by this method. This is also clear from the fact that piecewise linear test functions cannot take into account properly the singular behavior of the gradient of the solution to the continuous problem at the corner points. 
Acknowledgements. I wish to thank L. Malozemov for drawing my attention to the analysis on fractals and for checking the calculations. Part of this job was done at the Universities of Heidelberg and St.-Etienne and the author thanks Professors H. Rost and A. Bourgeat for hospitality and discussions.

\section{References}

1. Sierpinski, W.: Sur une courbe dont tout point est un point de ramification. C.R. Acad. Sci. Paris 160, 302 (1915)

2. Mandelbrot, B.B.: The fractal geometry of nature. New York: Freeman 1977

3. Rammal, R., Touluse, G.: Random walks on fractal structures and percolation clusters. J. Physique Lett. 44, L13-L22 (1983)

4. Halvin, S., Ben Avraham, D.: Diffusion in disordered media. Adv. Physics 36, 695-798 (1987)

5. Goldstein, S.: Random walks and diffusion on fractals. In: Kesten, H. (ed.): Percolation theory and ergodic theory of finite practical systems. IMA v. Math. Appl. 8. Berlin, Heidelberg, New York: Springer 1987, pp. 121-128

6. Kusuoka, S.: A diffusion process on a fractal. In: Itô, K., Ikeda, N. (eds.): Probabilistic methods in mathematical physics. Proc. Tanriguche Symp. Katata 1985. Amsterdam: Kunyo-North-Holland 1987, pp. 251-274

7. Barlow, M.T., Perkins, E.A.: Brownian motion on the Sierpinski gasket. Prob. Th. Rel. Fields 79, 543-623 (1988)

8. Lindstrøm, T.: Brownian Motion on nested fractals. Memoirs of the AMS 83, N420 (1990)

9. Barlow, M.T., Bass, R.F.: The construction of Brownian motion on the Sierpinski carpet. Ann. Inst. H. Poincaré 25, 225-257 (1989)

10. Matheron, G.: Eléments pour une théorie des milieux poreux. Masson et cie, Editeurs 120 Boulevard Saint-Germain, Paris, VIe (1967)

11. Zhikov, V.V., Kozlov, S.M., Oleinik, O.A.: Homogenization of differential operators: Berlin, Heidelberg, New York: Springer 1993

12. Kozlov, S.M.: Homogenization of random structures. Soviet Mathematics Doklady 19, N4, 950-954 (1987)

13. Kozlov, S.M.: On the duality of one type variational problems: Funkz. Anal. i ego Prilozhenija, 17/3, 9-15 (1983)

14. Bergman, D.J.: Bulk physical properties of composite media. In: Bergmann et al. (eds.): Les méthodes de l'homogénéisation: théorie et application en physique. Paris: Eyrolles 1985, pp. $1-128$

15. Golden, K., Papanicolaou, G.C.: Bounds for effective parameters of heterogeneous media by analytic continuation. Commun. Math. Phys. 90, 473-491 (1983)

16. Belyaev, A.Yu., Kozlov, S.M.: Hierarchical structures and estimates for homogenised coefficients. Russ. J. Math. Phys. 1, N 1, 3-15 (1992)

17. Julia, G.: Sur l'iteration des fonctions rationnelles. J. de Math. Pure et Appl. 8, 47-245 (1918)

18. Barlow, M.T., Bass, R.F.: On the resistance of the Sierpinski carpet. Proc. Roy. Soc. London Ser. A 431, N 1882, 345-360 (1990)

19. Kozlov, S.M., Vucan, Ya.: Explicit formula for thermoconductivity of quadratic lattice structure. C.R. Acad. Sci. Paris Ser. 1, 314, 281-286 (1992)

20. Poliya, G., Szego, G.: Aufgaben und Lehrsätze aus der Analysis. Berlin, Heidelberg, New York: Springer Vol. 1, 1964

Communicated by T. Spencer 
